\title{
Plasmonic Modes of Metamaterial-Based Slot Waveguides
}

\author{
Ivan D. Rukhlenko, ${ }^{1}$ Malin Premaratne, ${ }^{1}$ and Govind P. Agrawal ${ }^{2}$ \\ ${ }^{1}$ Advanced Computing and Simulation Laboratory (A $\chi$ L), Department of Electrical and Computer Systems Engineering, \\ Monash University, Clayton, VIC 3800, Australia \\ ${ }^{2}$ Institute of Optics, University of Rochester, Rochester, NY 14627, USA
}

Correspondence should be addressed to Ivan D. Rukhlenko, ivan.rukhlenko@monash.edu

Received 13 April 2012; Accepted 3 July 2012

Academic Editor: Alexandra E. Boltasseva

Copyright ( $) 2012$ Ivan D. Rukhlenko et al. This is an open access article distributed under the Creative Commons Attribution License, which permits unrestricted use, distribution, and reproduction in any medium, provided the original work is properly cited.

\begin{abstract}
Most metamaterials exhibit pronounced anisotropic properties that are crucial for the understanding of their superior optical behavior, especially when they are integrated into the structure of a plasmonic waveguide. In this paper, we analytically solve the dispersion relation for a slot plasmonic waveguide filled with an anisotropic-stratified metamaterial and reveal that it supports two modes featuring relatively long propagation lengths in the limit of vanishing slot thickness. We classify these modes according to their physical origin and study the variation of their dispersion properties with material parameters.
\end{abstract}

\section{Introduction}

The ultimate goal of optics is to enable a perfect control of the interaction between light and matter. This goal has been brought closer by the recent advances in nanotechnology that have made possible the fabrication of optical metamaterials $[1,2]$. The unusual electromagnetic properties of metamaterials are expected to enable a new generation of optical devices. In developing design strategies and new concepts for such devices, it is paramount that anisotropic properties of metamaterials are considered along with their other material features. Moreover, even the ways in which common devices operate require revisions when ordinary materials in their design are replaced by anisotropic metamaterials. A considerable amount of theoretical effort has been recently devoted to the analysis of optical propagation through different types of metamaterial structures, including uniaxial dielectrics [3] and indefinite media $[4,5]$, metal-dielectric heterostructures [6] and superlattices [7], and strongly anisotropic waveguides [8]. In this paper, we reexamine the guiding properties of slot plasmonic waveguides filled with an anisotropic medium. Our work is intended to demonstrate that integration of plasmonic waveguides with anisotropic optical metamaterials not only brings additional freedom to their design, but can also lead to new physical phenomena that may benefit the waveguide performance.
The plasmonic waveguide discussed here consists of an anisotropic medium of thickness $2 h$ embedded between two metals of permittivity $\varepsilon_{m}$. We assume that the medium's permittivity is described by a constant, diagonal tensor $\mathcal{\varepsilon}=\operatorname{diag}\left(\varepsilon_{x x}, \varepsilon_{y y}, \varepsilon_{z z}\right)$ with its principle axes parallel to the waveguide's edges. Even though the permeability has similar anisotropic properties, only one component of its tensor affects the transverse magnetic (TM) modes, which are of primary interest for plasmonic waveguides. This allows us to describe the permeability using a single parameter $\mu$. As is well known, the evolution of the electric field $\mathbf{E}=\left(E_{x}, 0, E_{z}\right)$ and the magnetic field $\mathbf{H}=\left(0, H_{y}, 0\right)$ of a TM mode is governed by the propagation constant $\beta$. In the case of an anisotropic core layer, $\beta$ obeys the dispersion relation

$$
\tanh (q h)=-\left[\left(\frac{k_{m}}{q}\right)\left(\frac{\varepsilon_{z z}}{\varepsilon_{m}}\right)\right]^{ \pm 1},
$$

where $q=\sqrt{\left(\varepsilon_{z z} / \varepsilon_{x x}\right) \beta^{2}-\varepsilon_{z z} \mu k^{2}}, k_{m}=\sqrt{\beta^{2}-\varepsilon_{m} k^{2}}, k=\omega / c$, $\omega$ is the frequency of the surface plasmon polariton (SPP), $c$ is the speed of light in vacuum, and the \pm signs correspond to the symmetric and antisymmetric modes, respectively.

Equation (1) is applicable to a broad range of metamaterial-based plasmonic waveguides and requires the specification of metamaterial design for further analysis. We restrict 


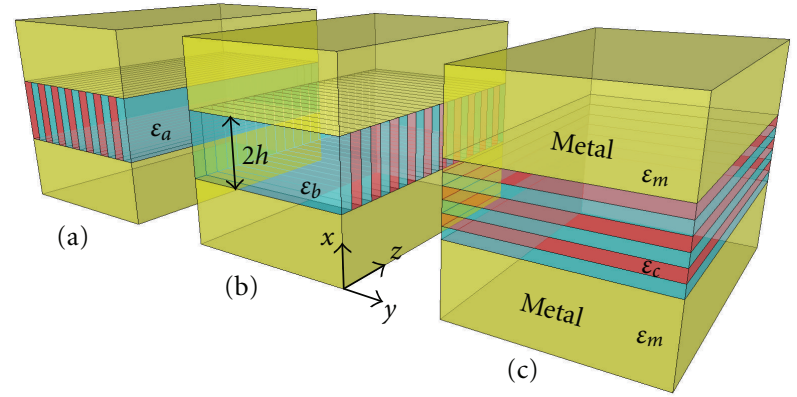

Figure 1: (Color online) Three types of plasmonic waveguides made using a stratified metamaterial. In all cases, SPP modes propagate in the $z$ direction and the waveguide extends to infinity in the $y$ direction.

ourselves to the simplest scenario in which the metamaterial is created via stacking layers of isotropic materials into a periodic heterostructure. If the layers' thicknesses are much smaller than the optical wavelength, then the permittivity tensor of the heterostructure has only two different diagonal components given by [9]

$$
\varepsilon_{\|}=\sum_{j=1}^{n} f_{j} \varepsilon_{j}, \quad \frac{1}{\varepsilon_{\perp}}=\sum_{j=1}^{n} \frac{f_{j}}{\varepsilon_{j}},
$$

where the subscripts $\|$ and $\perp$ designate directions parallel and perpendicular to the layers, $n$ is the number of layers within one heterostructure period, and $f_{j}$ is the filling factor for the $j$ th layer of permittivity $\varepsilon_{j}$ (all filling factors must add up to unity). Such a stratified metamaterial offers fabrication of the three types of plasmonic waveguides shown in Figure 1. Their permittivities are $\varepsilon_{a}=\operatorname{diag}\left(\varepsilon_{\|}, \varepsilon_{\perp}, \varepsilon_{\|}\right)$, $\varepsilon_{b}=\operatorname{diag}\left(\varepsilon_{\|}, \varepsilon_{\|}, \varepsilon_{\perp}\right)$, and $\varepsilon_{c}=\operatorname{diag}\left(\varepsilon_{\perp}, \varepsilon_{\|}, \varepsilon_{\|}\right)$. Although all of them can, in principle, be created using modern fabrication techniques, we focus here on the last structure that is relatively easy to fabricate.

Apart from the ease of fabrication, the third type of plasmonic waveguide in Figure 1 is the only one that supports symmetric TM modes with complex $\beta$ values in the limit of vanishing $\varepsilon_{\|}$, which can be realized by properly matching metamaterial constituents and compensating for the absorption losses inside them (note that the condition $\varepsilon_{\|}=0$ is not equivalent to an epsilon-near-zero (ENZ) regime [10], because the permittivity is a complex tensor). Within the slot region, these modes are characterized by the components $E_{x}$ and $H_{y}$ that are independent of $x$, and by $E_{z} \propto x$. Solving (1) in the limit $\varepsilon_{\|} \rightarrow 0$ yields the following four values of $\beta$ :

$$
\beta= \pm\left(\frac{1+2 r \mu K \pm \sqrt{1+4 r(\mu-r) K}}{2 r^{2} h^{2}}\right)^{1 / 2}
$$

where $K=\varepsilon_{m}(k h)^{2}$ and $r=\varepsilon_{m} / \varepsilon_{\perp}$ is the ratio of the dielectric constants. Since both of these parameters are generally complex, $\beta=\beta^{\prime}+i \beta^{\prime \prime}$ is also complex, and its real and imaginary parts provide the phase velocity and energy loss of various SPP modes. Because $\beta$ in (3) satisfies (1) approximately in the limit of small $h$ (when $|q| h \ll 1$ ) even when $\varepsilon_{\|} \neq 0$, the following analysis allows us to elucidate some general features of the SPP behavior in metamaterialbased plasmonic waveguides.

For simplicity, we focus on the stratified heterostructure composed of two different nonmagnetic $(\mu=1)$ materials, one of which is the metal used for waveguide cladding. The permittivity of the second material is then fixed by the condition $\varepsilon_{\|}=0$ (this material should provide gain, since metals are essentially lossy). It then follows that the ratio $r$ depends solely on the filling factor $f$ of the metal; $r=$ $2-1 / f$. We use silver as a metal in our simulations, take its permittivity in the form of a seven-pole Drude-Lorentz formula given in the work by Pannipitiya et al. [11], and introduce an absorption parameter $\gamma$, such that $\varepsilon_{m}(\gamma)=\varepsilon^{\prime}+$ $i \gamma \mathcal{E}^{\prime \prime}$. For definiteness, we also choose $\beta^{\prime}>0$ and refer to $\beta^{\prime \prime}$ as the "damping factor," thus implying that our waveguides do not amplify SPPs.

According to (3), the waveguide in Figure 1(c) supports a maximum of two SPP modes regardless of slot's thickness, provided that $\varepsilon_{\|}=0$. The dispersion curves of these two modes are plotted in Figure 2 for three values of $\gamma$ and two sets of material parameters $f$ and $h$. In the lossless case ( $\gamma=0$, green curves), the modes can be grouped into three distinct classes: (i) propagating modes with $\beta^{\prime \prime}=0$ and $\beta^{\prime} \neq 0$; (ii) complex modes with $\beta^{\prime \prime} \neq 0$ and $\beta^{\prime} \neq 0$; (iii) evanescent modes with $\beta^{\prime \prime} \neq 0$ and $\beta^{\prime}=0$. The first two types of modes may travel either forward or backward, except for certain "degenerate" frequencies for which the group velocity and total energy flow of SPPs vanish. In our example, the degenerate frequencies correspond to the points $\mathrm{A}, \mathrm{B}$, and $\mathrm{C}$ of the energy spectrum. The degeneracy of waves traveling in the opposite directions is removed by absorption (see pink curves for $\gamma=0.2$ ) since the two modes develop a slightly different lateral confinement.

The complex modes of a lossy waveguide arise through quasimixing of the three types of modes as $\gamma$ is gradually varied from 0 to 1 . Such transformation of the propagating, complex, and/or evanescent modes is accompanied by their degeneracy removal, which is also the scenario followed by the quadrupole modes of plasmonic nanowires [12]. Indeed, the twofold degenerate propagating mode at point B and the twofold degenerate evanescent mode at point $\mathrm{C}$ split into two nondegenerate complex modes for $\gamma=1$ (see Figure 2). The process of modes transformation allows one to track their 


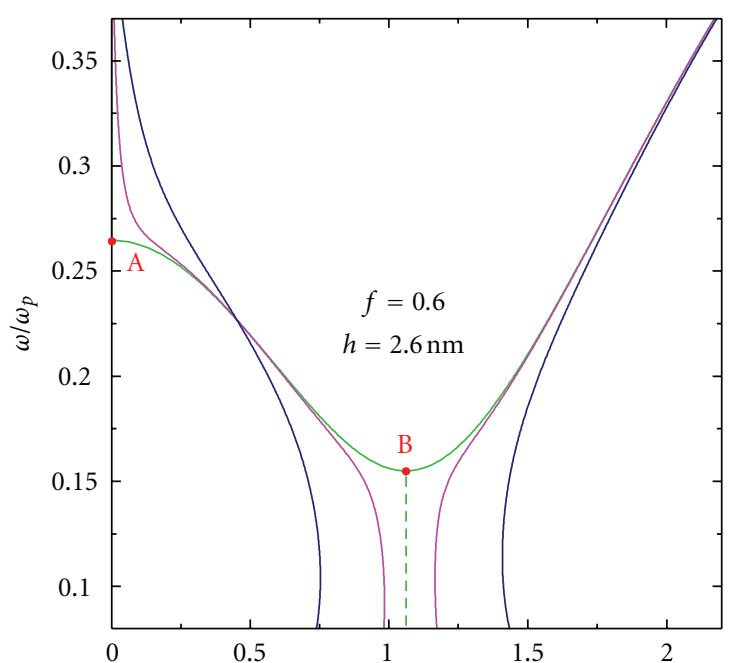

(a)

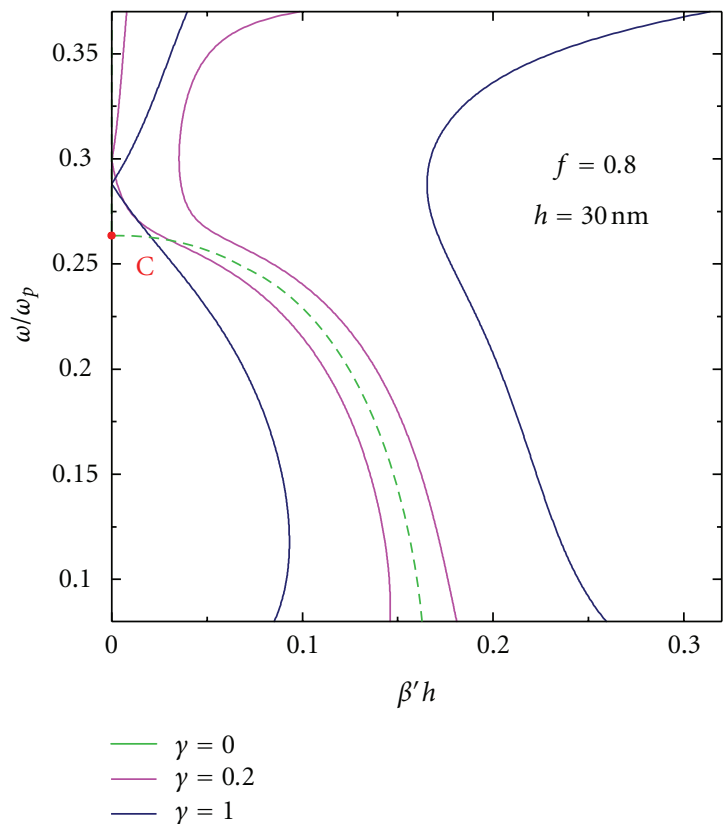

(c)

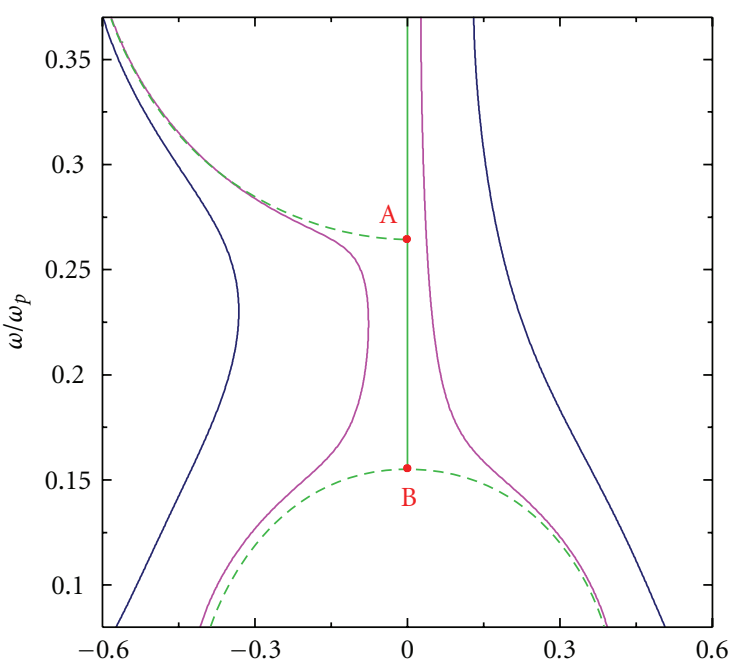

(b)

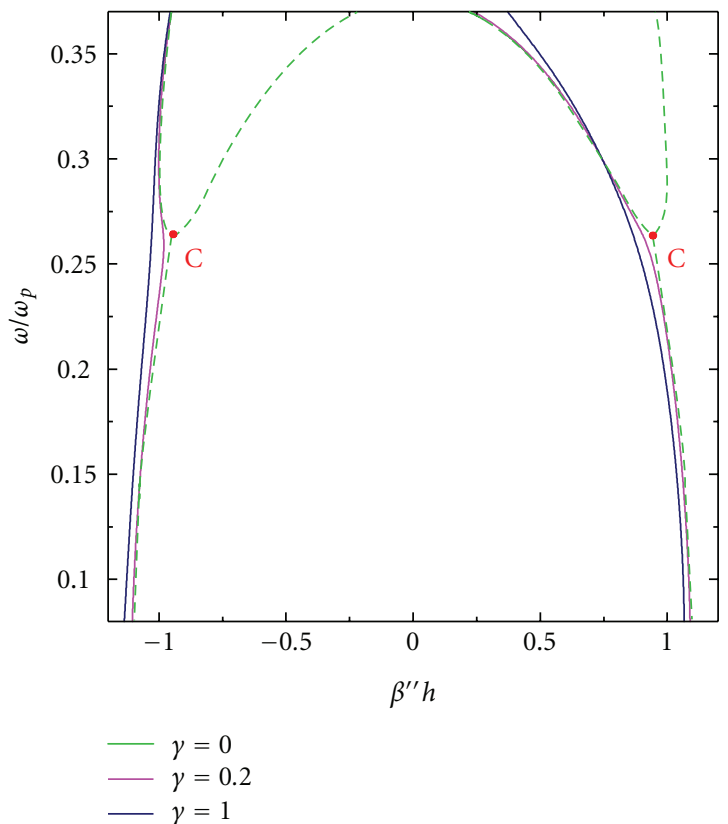

(d)

Figure 2: (Color online) Transformation of [(a) and (c)] SPP energy spectrum and [(b) and (d)] damping-factor dispersion with changing absorption parameter $\gamma$. The SPP frequency is normalized to the plasma frequency $\omega_{p}$ of silver. Solid green curves show propagating modes, whereas dashed curves show complex and evanescent modes.

origin and better understand waveguide performance. For example, because of the strong damping intrinsic to evanescent modes, one can expect that the segments of spectra $\beta^{\prime \prime}(\omega)$ emerging from them in the case of real losses will be lesser affected by the dispersion properties of $\varepsilon^{\prime \prime}(\omega)$ than the segments that stem from undamped propagating modes. This conclusion is confirmed by Figures 2(b) and 2(d), where the damping factors are separately shown for the forward (right curves) and backward (left curves) propagating SPP modes. Notice that the spectra in Figure 2 are congregate of the results valid for different plasmonic waveguides, rather than spectra corresponding to a specific structure.

As we have seen, dispersions of the real and imaginary parts of $\beta$ drastically depend on the filling factor of metal and slot thickness. To study this dependance, we set $\omega=$ $0.2 \omega_{p}$, where $\omega_{p}$ is the plasma frequency of silver, and plot in Figure $3 \beta^{\prime}$ and $\beta^{\prime \prime}$ as functions of slot thickness for five values of $f$. By looking at this figure from left to right, one can draw up the following picture of SPP behavior. When the filling factor of metal is small, two SPP modes of different 

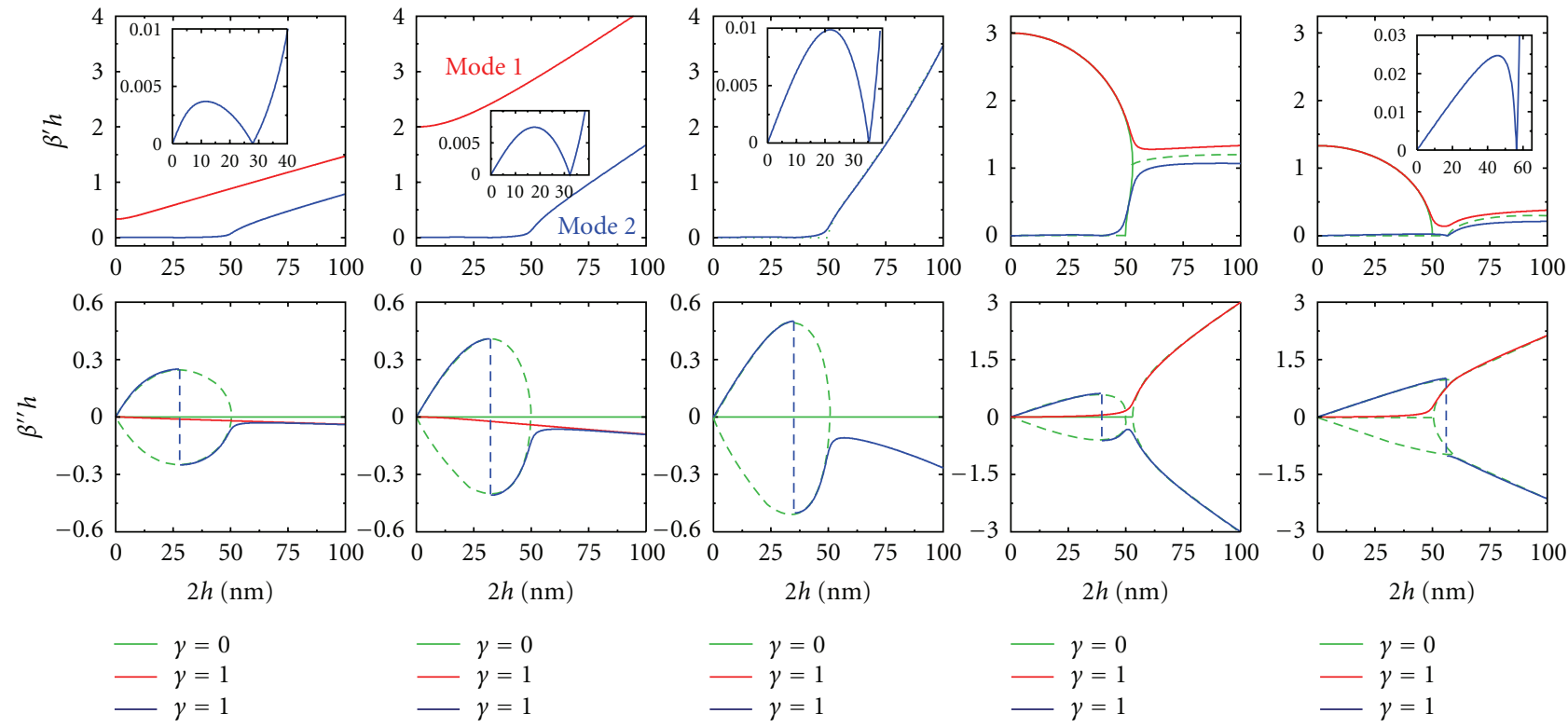

$\begin{aligned} \gamma & =1 \\ -\gamma & =1\end{aligned}$
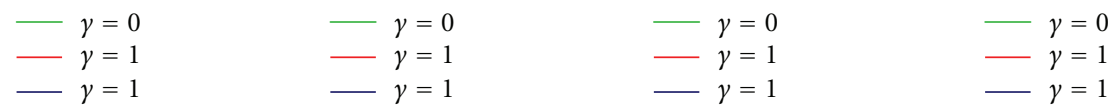

(a) $f=0.2$

(b) $f=0.4$

(c) $f=0.5$

(d) $f=0.6$

(e) $f=0.8$

FIgURE 3: (Color and multimedia online) Transformation of the real (upper panels) and imaginary (lower panels) parts of $\beta$ at a specific frequency $\omega=0.2 \omega_{p}$ as the filling factor $f$ of the metal is increased from 0.2 to 0.8 . Insets show a magnified view of mode 2 .

origins are supported by the waveguide. One of themmode 1 shown by red curves - springs from the propagating mode of a lossless waveguide and, therefore, exhibits small damping. The other-mode 2 plotted in blue-arises from the evanescent mode when $h<h_{0}=k^{-1}\left|\varepsilon_{m}\right|^{-1 / 2} \approx 25 \mathrm{~nm}$ and from the propagating mode when $h>h_{0}\left(h_{0}\right.$ is the skin depth of a metal in the limit $f \rightarrow 0$ ). The first mode propagates backward regardless of $h$, whereas the second one changes its traveling direction from forward to backward around $h=h_{0} / 2$. Because modes 1 and 2 originate from the propagating modes for $h>h_{0}$ and $f<0.5$, their damping rates are almost the same for this range of parameters $h$ and $f$. When $f=0.5$, the ratio $r=0$ since the transverse component of the permittivity tensor becomes infinite, and only mode 2 with $\beta= \pm k \sqrt{\varepsilon_{m}(1+K)}$ survives. For $f>0.5$, mode 1 becomes forward propagating, while mode 2 gets strongly attenuated for all $h$. The overall dynamics of such changes with $f$ is illustrated in the supplementary animation. It should be emphasized that these results are based on the effective-medium theory and approximate. For a more accurate treatment, the inclusion of nonlocality effects is required $[13,14]$.

An important feature of the modes given in (3) is that they can exhibit relatively small damping depending on the values of the parameters $f$ and $h$. For example, low damping occurs for both modes for $f<0.2$ and $h>h_{0}$. Moreover, the damping of mode 1 vanishes in the limit of small $h$ for all values of $f \neq 0.5$. This behavior is opposite to that of SPPs in ordinary (isotropic) metal-dielectric-metal waveguides, whose attenuation rate diverges as $1 / h[15]$. Also noteworthy is that the confinement of mode 1 , determined by the evanescent decay length scaling like $\delta_{1} \propto h \operatorname{Re} \sqrt{r^{2}}$ when $h \rightarrow 0$, improves with the reduction of the waveguide thickness, whereas the confinement of mode 2 worsens in the limit of small $h$, due to the divergence of the decay length $\delta_{2} \propto$

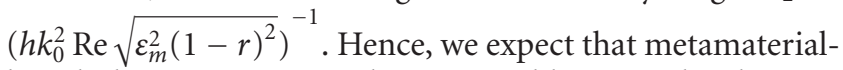
based plasmonic waveguides may enable strong localization of optical energy in an SPP mode that can propagate over relatively long lengths.

In conclusion, we have found that a maximum of two symmetric TM modes with essentially different propagation properties are supported by plasmonic slot waveguides filled with a stratified metamaterial exhibiting a relatively small permittivity in the plane of the layers.

\section{Acknowledgments}

This work was supported by the Australian Research Council, through its Discovery Grant scheme under Grant DP110100713 and by the Discovery Early Career Researcher Award DE120100055.

\section{References}

[1] W. Cai and V. Shalaev, Optical Metamaterials: Fundamentals and Applications, Springer, New York, NY, USA, 2010.

[2] V. G. Veselago and E. E. Narimanov, "The left hand of brightness: past, present and future of negative index materials," Nature Materials, vol. 5, no. 10, pp. 759-762, 2006.

[3] P. A. Belov, "Backward waves and negative refraction in uniaxial dielectrics with negative dielectric permittivity along the anisotropy axis," Microwave and Optical Technology Letters, vol. 37, no. 4, pp. 259-263, 2003. 
[4] D. R. Smith, D. Schurig, J. J. Mock, P. Kolinko, and P. Rye, "Partial focusing of radiation by a slab of indefinite media," Applied Physics Letters, vol. 84, no. 13, pp. 2244-2246, 2004.

[5] D. R. Smith and D. Schurig, "Electromagnetic wave propagation in media with indefinite permittivity and permeability tensors," Physical Review Letters, vol. 90, no. 7, Article ID 077405, pp. 1-4, 2003.

[6] J. Elser, V. A. Podolskiy, I. Salakhutdinov, and I. Avrutsky, "Nonlocal effects in effective-medium response of nanolayered metamaterials," Applied Physics Letters, vol. 90, no. 19, Article ID 191109, 2007.

[7] S. M. Vukovic, I. V. Shadrivov, and Y. S. Kivshar, "Surface bloch waves in metamaterial and metal-dielectric superlattices," Applied Physics Letters, vol. 95, no. 4, Article ID 041902, 2009.

[8] V. A. Podolskiy and E. E. Narimanov, "Strongly anisotropic waveguide as a nonmagnetic left-handed system," Physical Review B, vol. 71, no. 20, Article ID 201101, 4 pages, 2005.

[9] I. D. Rukhlenko, M. Premaratne, and G. P. Agrawal, "Theory of negative refraction in periodic stratified metamaterials," Optics Express, vol. 18, no. 26, pp. 27916-27929, 2010.

[10] A. Alù, M. G. Silveirinha, A. Salandrino, and N. Engheta, "Epsilon-near-zero metamaterials and electromagnetic sources: tailoring the radiation phase pattern," Physical Review B, vol. 75, no. 15, Article ID 155410, 2007.

[11] A. Pannipitiya, I. D. Rukhlenko, M. Premaratne, H. T. Hattori, and G. P. Agrawal, "Improved transmission model for metaldielectric-metal plasmonic waveguides with stub structure," Optics Express, vol. 18, no. 6, pp. 6191-6204, 2010.

[12] A. R. Davoyan, W. Liu, A. E. Miroshnichenko, I. V. Shadrivov, Y. S. Kivshar, and S. I. Bozhevolnyi, "Mode transformation in waveguiding plasmonic structures," Photonics and Nanostructures, vol. 9, no. 3, pp. 207-212, 2011.

[13] A. A. Orlov, P. M. Voroshilov, P. A. Belov, and Y. S. Kivshar, "Engineered optical nonlocality in nanostructured metamaterials," Physical Review B, vol. 84, no. 4, Article ID 045424, 2011.

[14] A. V. Chebykin, A. A. Orlov, A. V. Vozianova, S. I. Maslovski, S. Yu. Kivshar, and P. A. Belov, "Nonlocal effective medium model for multilayered metal-dielectric metamaterials," Physical Review B, vol. 2011, Article ID 115438, 2011.

[15] I. D. Rukhlenko, M. Premaratne, and G. P. Agrawal, "Nonlinear propagation in silicon-based plasmonic waveguides from the standpoint of applications," Optics Express, vol. 19, no. 1, pp. 206-217, 2011. 

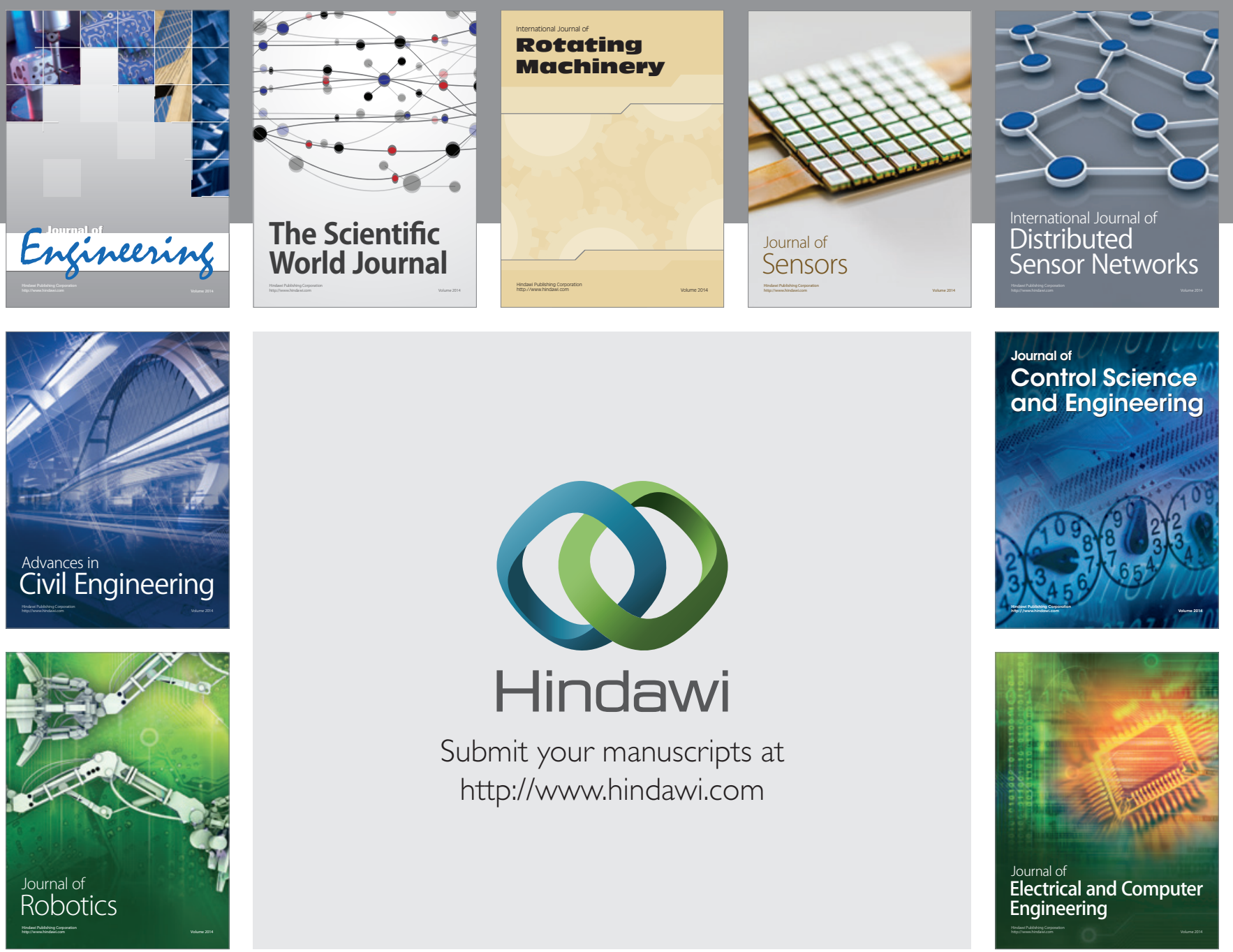

Submit your manuscripts at

http://www.hindawi.com
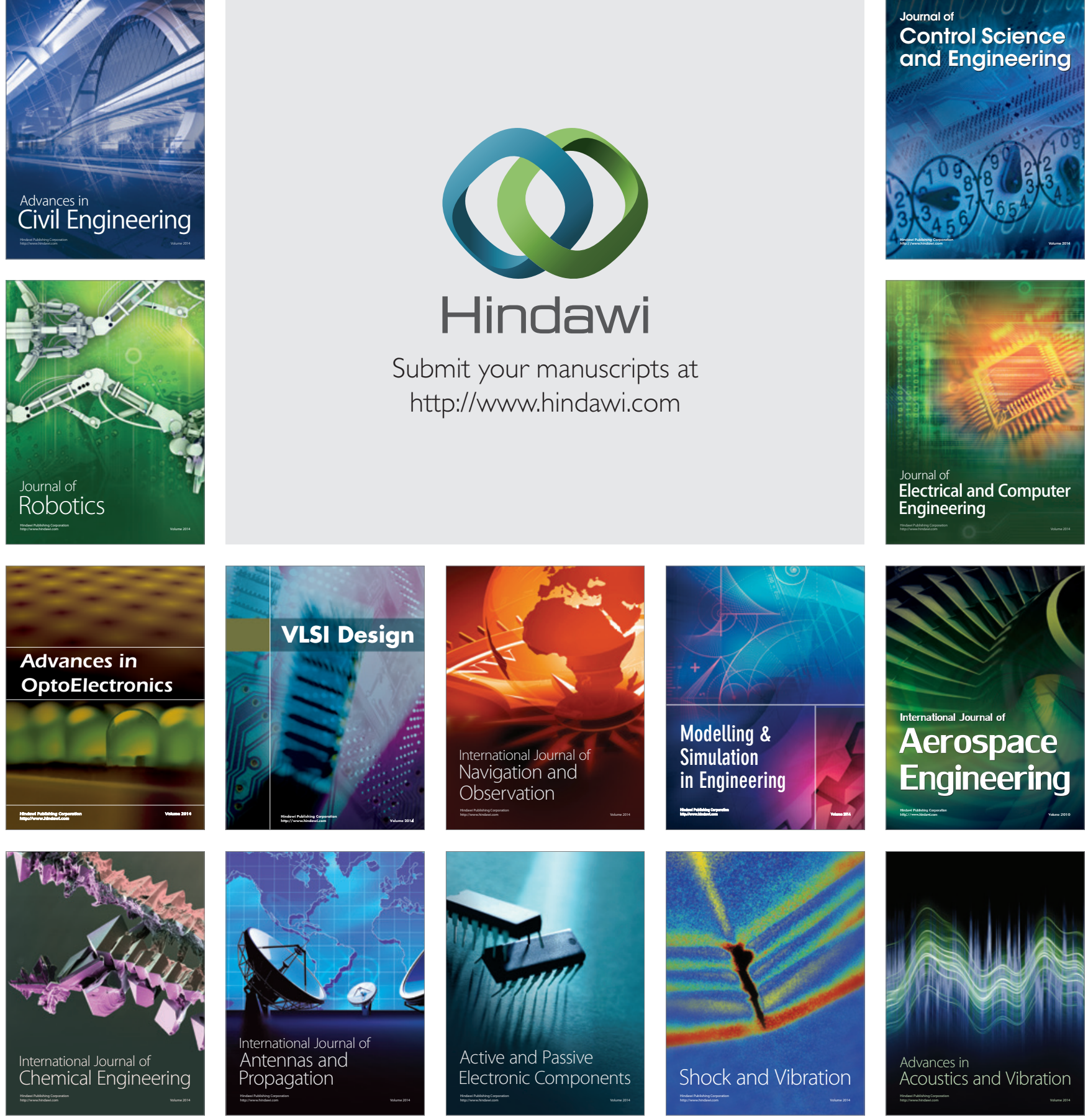